\title{
Imaging the Statics and Dynamics of Superconducting Vortices and Antivortices Induced by Magnetic Microdisks
}

\author{
R. B. G. Kramer, ${ }^{1,2}$ A. V. Silhanek, ${ }^{1,3}$ W. Gillijns, ${ }^{1}$ and V. V. Moshchalkov ${ }^{1}$ \\ ${ }^{1}$ INPAC, Institute for Nanoscale Physics and Chemistry, Nanoscale Superconductivity and Magnetism Group, K. U. Leuven, \\ Celestijnenlaan 200D, B-3001 Leuven, Belgium \\ ${ }^{2}$ Institut Néel, CNRS, Université Joseph Fourier, BP 166, 38042 Grenoble Cedex 9, France \\ ${ }^{3}$ Département de Physique, Université de Liège, B- 4000 Liège, Belgium
}

(Received 20 May 2011; published 17 October 2011)

\begin{abstract}
If a magnet of microscopic dimensions is brought in close proximity to a superconductor, the quantized nature of their interaction due to the creation of flux quanta in the superconducting system becomes noticeable. Herein, we directly image, via scanning Hall microscopy, the vortex-antivortex pairs in a superconducting film created by micromagnets. The number of antivortices at equilibrium conditions can be changed either by tuning the magnetic moment of the magnets or by annihilation with externally induced vortices. We demonstrate that small ac field excitations shake the antivortices sitting next to the micromagnets whereas no sizable motion is observed for the vortices sitting on top of the magnets, clearly revealing the different mobility of these two vortex species. A metastable state, which is obtained by applying a field after the system has been cooled down below the superconducting transition, shows a complex graded distribution of coexisting vortices and antivortices forming an intertwined critical state.
\end{abstract}

DOI: 10.1103/PhysRevX.1.021004

Subject Areas: Superconductivity

\section{INTRODUCTION}

Superconductors differ from perfect conductors by their lossless diamagnetic nature, which arises from the persistent screening currents minimizing the internal magnetic field. If a ferromagnetic particle with a net dipolar magnetic moment and dimensions much smaller than the superconductor approaches the latter, an image of the magnetic particle appears within the superconductor as a consequence of the boundary conditions [1]. This image magnet generates a repelling force on the magnetic particle which can make the particle levitate. However, if the magnetic dipole is forced to stay closer to the superconducting material than the equilibrium levitation height, their stray field may become strong enough to penetrate into the bulk of the superconductor.

The inevitable transformation of the magnet's continuous stray field outside the superconductor into quantum flux-line bundles inside the superconductor gives rise to several interesting properties such as topological instabilities in superconductor-ferromagnet bilayers [2], field-induced superconductivity [3], domain-wall superconductivity [4], composite self-organized critical states $[5,6]$, or quantized levitation height [7,8]. It is also crucial to take into consideration the interaction of quantized flux lines with ferromagnets in order to understand more practical systems such as magnetic force microscopy measurements of superconducting samples $[9,10]$, the enhancement of

Published by the American Physical Society under the terms of the Creative Commons Attribution 3.0 License. Further distribution of this work must maintain attribution to the author(s) and the published article's title, journal citation, and DOI. the superconducting critical current in superconductorferromagnet hybrid systems [11-14], or why superconducting levitating trains do not derail in sharp curves $[15,16]$.

Most of the work so far has focused on the theoretical aspects of these systems with little direct experimental evidence of the static behavior [17] of the induced vortex-antivortex (VAV) pairs, and even much less of their dynamics. This is so, in part due to the difficulties to resolve, at submicrometer scales, closely packed VAV pairs, which tend to severely reduce the stray fields. In this paper, we combine two imaging techniques with stateof-the-art single-vortex resolution, in order to investigate the conditions for formation of VAV pairs, their interaction with an external field, and the mobilities of different vortex species.

We first illustrate how magnetic disks with zero average magnetic moment, which do not generate VAV pairs, act as efficient traps for externally induced vortices of any polarity. Then, by slightly magnetizing the disks perpendicular to the plane of the superconducting film, it is possible to break the field-polarity symmetry in such a way that flux lines with opposite polarity than the magnetic moment of the disks (i.e., antivortices) are repelled by them, whereas the disks become attractors for flux lines with the same polarity than the magnetic moment of the disks (i.e., vortices). Further increasing the average magnetic moment of the disks, VAV pairs are spontaneously created, with a number of AV (or V) that depends on temperature, external field, and net magnetic moment. We also explore the nonequilibrium properties of the flux distribution and observe a complex flux pattern with coexisting gradients of vortices and antivortices for strongly magnetized disks. Finally, by means of a scanning acsusceptibility technique, we are able to visualize for the first 
time the contrasting mobilities of vortices sitting on top of the magnetic disks, with respect to the antivortices lying next to them.

\section{EXPERIMENTAL DETAILS AND SAMPLE LAYOUT}

To carry out our studies, we prepared, via electron beam lithography and subsequent molecular beam epitaxy deposition, a square periodic array of Co-Pt circular disks of $1 \mu \mathrm{m}$ diameter, $20 \mathrm{~nm}$ thickness, and $5 \mu \mathrm{m}$ separation, spanning an area of $5 \times 5 \mathrm{~mm}^{2}$ in a $10 \times 10 \mathrm{~mm}^{2} \mathrm{Si}$ substrate. Each ferromagnetic disk consists of a $2.5 \mathrm{~nm}$ Pt buffer layer covered with a $(0.4 \mathrm{~nm} \mathrm{Co} / 1.0 \mathrm{~nm} \mathrm{Pt})_{10}$ multilayer with magnetization perpendicular to the sample surface [18]. A $50 \mathrm{~nm}$ thick superconducting $\mathrm{Pb}$ film with critical temperature $T_{c}=7.2 \mathrm{~K}$ was evaporated on top, covering the whole substrate, i.e., the patterned area with magnetic disks and all the surrounding unpatterned region. This permits us to examine the field distribution in a region without magnetic disks and more important, to determine with a very high accuracy the zero effective field. Figure 1 shows a schematic representation of the sample layout. In order to avoid the suppression of superconductivity on top of the disks by exchange interaction, a $5 \mathrm{~nm}$ thick $\mathrm{Ge}$ insulating layer separates the disks from the $\mathrm{Pb}$ film. The substantial difference between the range of explored fields $(|H|<10 \mathrm{mT})$ and the coercive field of the ferromagnet $\left(H_{c} \sim 50 \mathrm{mT}\right)$ guarantees no influence of the superconductor on the magnetic state of the disks. Full saturation of the disks is achieved at the $800 \mathrm{mT}$ perpendicular field, producing a magnetic moment $M_{s}=4.47 \times 10^{5} \mathrm{~A} / \mathrm{m}$. The average remanent magnetization of the disks can be

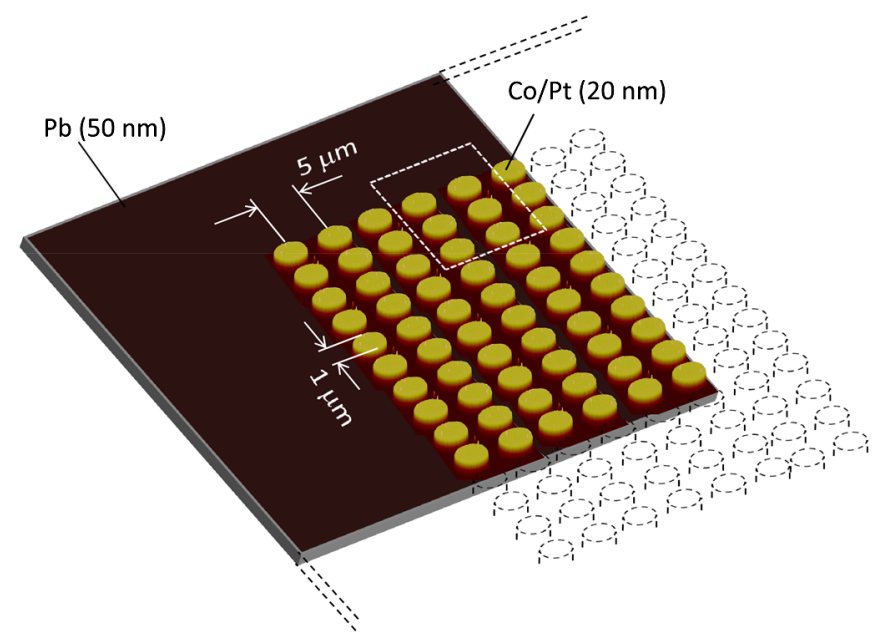

FIG. 1. Schematic representation of the sample layout. Co-Pt disks $20 \mathrm{~nm}$ thick and of $1 \mu \mathrm{m}$ diameter forming a $5 \mu \mathrm{m}$ period square array which covers partially a Si substrate. A type-II superconducting Pb film $50 \mathrm{~nm}$ thick covers the whole substrate including the magnetic disks. The rectangular area delimited by a dashed white line indicates the typical scanning area investigated via Hall microscopy. reversibly controlled by following a careful degaussing procedure as explained in Ref. [19].

The scanning Hall-probe microscopy images are obtained using a modified low-temperature scanning Hallprobe microscope from Nanomagnetics Instruments. The Hall probe with a size of $600 \times 600 \mathrm{~nm}^{2}$ is structured in the two-dimensional electron gas of a GaAs or AlGaAs heterostructure and has a sensitivity of $6 \times 10^{-2} \mathrm{~V} / \mathrm{T}$ with an applied dc current of $25 \mu \mathrm{A}$. The scan range is $14 \times 14 \mu \mathrm{m}^{2}$ at $4.2 \mathrm{~K}$ and $128 \times 128$ pixels are recorded with a scan speed of $60 \mu \mathrm{m} / \mathrm{s}$. The sample-probe distance is controlled with a gold electrode (STM tip) patterned approximately $15 \mu \mathrm{m}$ from the center of the Hall probe. The Hall probe is mounted with a small tilt angle of approximately $3^{\circ}$ with respect to the sample surface. The STM tip is lifted off by $200 \mathrm{~nm}$ from the sample surface, which results in a total Hall-probe or sample distance of typically $\sim 0.7 \mu \mathrm{m}$. The scan plane is adjusted to be parallel to the sample surface. Furthermore, piezo motors allow for a sample coarse positioning in a range of $3 \times 3 \mathrm{~mm}^{2}$.

\section{MULTIVORTEX TRAPPING OF DEMAGNETIZED DISKS}

Owing to the large diameter of the disks, both the asgrown state and the fully demagnetized state microscopically correspond to a magnetic multidomain state with a very localized (high order multipole) stray field. Yet this local field is sufficient to deplete the superconducting condensate and favor the vortex trapping [20]. Figures 2(a)-2(d) show (a)

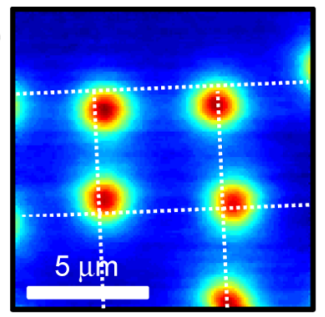

(c)

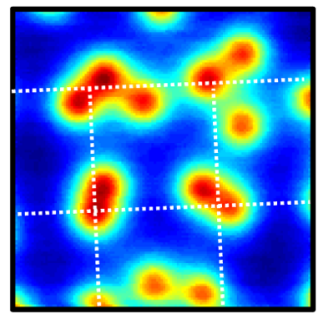

(b)

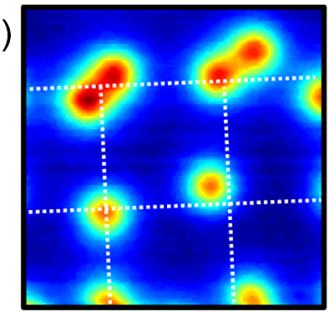

(d)

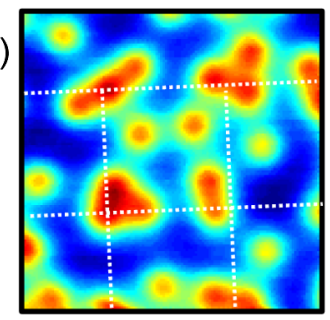

FIG. 2. Trapping of single-quantum vortices by demagnetized disks. (a)-(d) Scanning Hall microscopy image at the border of the patterned area, obtained at $T=4.2 \mathrm{~K}$. The white dashed lines connect nearest-neighbor magnetic disks. (a) Individual vortices at $H=0.7 H_{1}$. (b) Coexistence of individual vortices and vortex dimers at $H=1.2 H_{1}$. (c) Coexistence of vortex dimers and trimers $H=2.1 H_{1}$. (d) Different vortex trimers at $H=3.4 H_{1}$ coexisting with interstitial vortices. 
scanning-Hall-probe-microscopy (SHPM) images, obtained at $T=4.2 \mathrm{~K}$, evidencing the formation of vortex clusters on top of the magnetic disks in the demagnetized state. Since the SHPM technique is sensitive to the local field approximately $700 \mathrm{~nm}$ above the sample's surface, the magnetic disks in the demagnetized state with a little stray field become difficult to discern at low temperatures due to the substantial screening produced by the Meissner supercurrents. In this case, a scan above the superconducting critical temperature is necessary to unveil the position of the magnets.

In Fig. 2 the positions of the disks coincide with the intersections of the dashed lines. By applying an external field above the superconducting critical temperature $T_{c}$ and subsequently cooling down, vortices nucleate in the close vicinity of the disks. For low fields, individual single-quantum vortices are trapped by the micromagnets [Fig. 2(a)]. Increasing the field above the first commensurability field $H_{1}=0.0828 \mathrm{mT}$, dimers or trimers of vortices agglomerate around the magnetic disks. The fact that interstitial vortices appear once the trimers are formed indicates a saturation number, i.e., a maximum number of trapped vortices, of 3 . As we will discuss below, this saturation number depends on the total magnetic moment of the disks. Images similar to the one shown in Fig. 2 are obtained upon reversing the field polarity. However, once the disks acquire a net magnetic moment the field-polarity symmetry is no longer sustained. This is illustrated in Figs. 3(a)-3(c) where the disks have a magnetic moment $M=+0.75 M_{s}$ and five scanning areas were stitched together, spanning from outside to deep inside the patterned region. The flux distribution at zero field [Fig. 3(b)] shows the coexistence of vortices (red spots) and antivortices (blue spots). Notice that the vortices sit on top of the disks whereas the antivortices tend to avoid them [21,22]. In other words, the field-polarity symmetry has been broken by the directional moment of the disks. This is in agreement with what is predicted by the electromagnetic-interaction energy between a magnetic dipole and a vortex line within the London formalism [23],

$$
U_{p}(\mathbf{R})=-\int_{d o t} \mathbf{m}(\mathbf{r}) \cdot \mathbf{B}_{v}(\mathbf{R}-\mathbf{r}) d^{3} r,
$$

where the integration is carried out over the volume of the ferromagnet, $\mathbf{R}$ indicates the position of the vortex line, $\mathbf{m}(\mathbf{r})$ its spatial dependent magnetic moment, and $\mathbf{B}_{v}$ is the field generated by the vortex line.

It is interesting to note that in the zero-field image shown in Fig. 3(b), there are 5 antivortices and 2 vortices within an area of about $676 \mu \mathrm{m}^{2}$, corresponding to a magnetic field of $H \sim 0.009 \mathrm{mT}$. This is basically the field step in between consecutive field cooling used to determine the zero-field condition and justifies the observed unbalanced distributions of vortices and antivortices. However, it seems intriguing that at zero field we have vortices and antivortices rather than no vortices at all. A plausible explanation for this behavior is that the magnetic disks with a magnetic moment $M=+0.75 M_{s}$, even though they are unable to induce vortex-antivortex pairs at low temperatures, may generate vortex-antivortex pairs close to the superconducting critical temperature. Within this scenario, during a cooling procedure at a nonzero field most of the vortex-antivortex pairs induced close to $T_{c}$ annihilate (a)

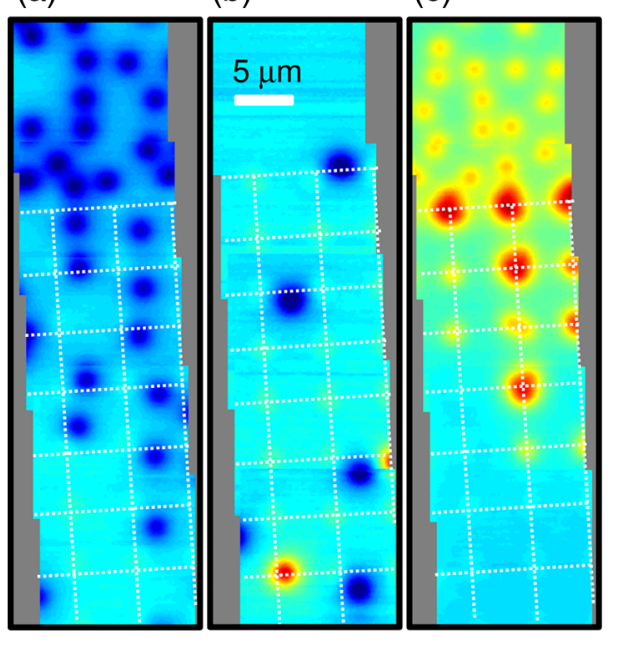

(d)

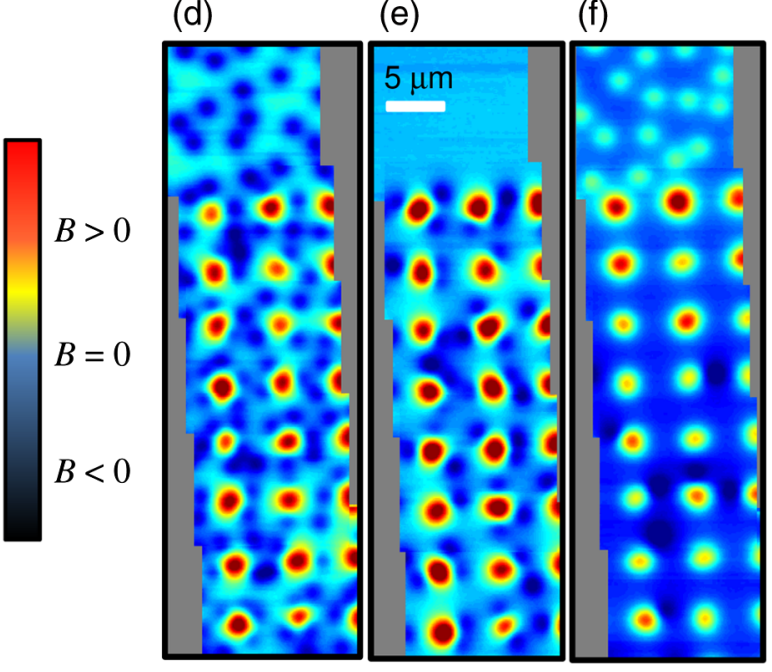

FIG. 3. Nonequilibrium flux penetration from the border of the pattern. (a)-(f) Stitching of several Scanning Hall microscopy images showing the flux distribution from outside to inside the patterned area after the field has been increased at $T=7 \mathrm{~K}$. (a)-(c) Magnetic disks with a small magnetic moment pointing out of the page. (a) $H=-0.785 H_{1}$. (b) $H=0$. (c) $H=+0.785 H_{1}$. Flux lines with the same polarity as the disks (vortices) penetrate less than flux lines with opposite polarity as the disks (antivortices). (d)-(f) Magnetic disks with a large magnetic moment such that vortex-antivortex pairs are induced at zero external field (e). The annihilation of antivortices occurs for positive fields (f) $H=+1.57 H_{1}$ whereas at negative fields (d) $H=-2.3 H_{1}$ extra antivortices are externally added. The color bar indicates the intensity of the local field measured by the Hall probe: black for negative fields and red for positive fields. 


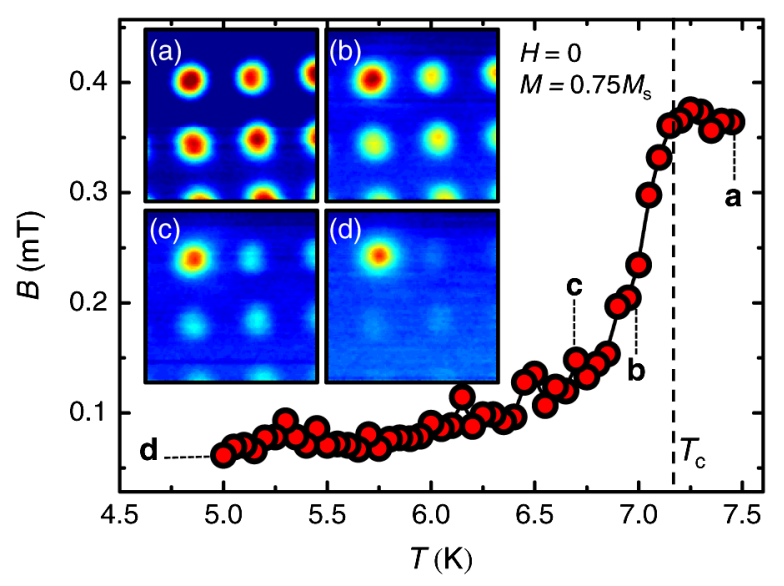

FIG. 4. Supercurrents screening the stray field of the micromagnets. (a)-(d) Scanning Hall-probe microscopy pictures at the same spot on the pattern for progressively decreasing temperature as indicated in the main panel. At the lowest temperature (d), most of the stray field does not penetrate into the superconductor. The main panel shows the average positive field in the scanning area as a function of temperature.

themselves as the temperature decreases but a minority of them may survive in a metastable state. This interpretation is supported by the fact that in Fig. 3(b), (i) vortexantivortex pairs appear only within the patterned area and (ii) each vortex has an antivortex in the vicinity (i.e., within a unit cell distance). In order to obtain more conclusive evidence, we recorded images, while cooling the sample down in zero external field, in an area near the center of the sample and for the disks with magnetic moment $M=+0.75 M_{s}$. The results are summarized in Fig. 4 .

The main panel of Fig. 4 shows the average magnetic field in the scanning area as a function of temperature. For each point in the main panel there is a corresponding SHPM image. In the insets Figs. 4(a)-4(d) we show representative SHPM images at the temperatures indicated in the main panel. For temperatures above the superconducting transition, the magnetic disks are clearly visible and allow one to unequivocally identify their position. As the temperature drops below $T_{c}$ the intensity of the field on top of the disks fades rapidly for three of them whereas the remaining disk retains a vortex-antivortex pair. This indicates that for this particular magnetic moment, i.e., close to the generation of a vortex-antivortex pair at low temperatures, some vortexantivortex pairs may appear even at zero external field.

\section{COMPLEX VORTEX PENETRATION FOR MAGNETIZED DISKS}

The field-polarity-dependent vortex pinning discussed above becomes more clear by comparing Figs. 3(a) and 3(c), which show the field penetration (i.e., increasing the field after the system has been cooled down) for the same field but opposite polarity. For negative fields [Fig. 3(a)], antivortices are repelled by the magnetic disks, thus favoring their channeling inside the patterned area. In contrast to that, vortices induced by a positive field [Fig. 3(c)] get trapped by the magnetic disks and penetrate less into the patterned region. It is worth noticing that in the latter case, multiquanta vortices appear at the border of the pattern due to the high saturation number of the micromagnets.

A different situation emerges when the magnetic disks have a higher magnetic moment of $M=0.99 M_{s}$ as shown in Figs. 3(d)-3(f). Indeed, the strong stray field emanating from the disks can now generate either multiquanta giant vortices or a tightly confined multivortex bundle on top of the disks, which, due to flux conservation, split into singlequantum antivortices surrounding them [24]. This is more clearly seen in Fig. 3(e) obtained at zero external field. In this case, it is unnecessary to indicate the position of each disk since it is revealed by the vortices sitting on top (red spots). The smaller dark blue spots around the disks correspond to the antivortices that average to three per disk. In agreement with previous indirect measurements, the actual number of vortex-antivortices pairs depends not only on the magnetic moment of the disks but also on their geometry [17,24,25]. In Ref. [17], for Co-Pt disks of similar size to the ones investigated here but having more magnetic material (larger number and slightly thicker of Co layers), the authors showed that 5 vortex-antivortex pairs are created at saturation, likely due to the higher remanent magnetic moment. It is also important to note that in Fig. 3(e) the antivortices do not form a regular array, but rather cluster around the magnetic disks [26]. This is in agreement with theoretical predictions by Milošević and Peeters, showing that for large periods of the disk array, the vortex-antivortex pairs arrange similarly to isolated, i.e., no interacting, disks [27].

The question now arises as to whether the vortices on top of the disks merge into a giant multiquanta vortex or remain as separated single-quantum units. The fact that we are unable to resolve individual vortices on top of the disks should not be viewed as an evidence of giant vortices because of the limited spatial resolution of our scanning Hall microscope $(\sim 0.5 \mu \mathrm{m})$. According to the theoretical calculation in Ref. [24], it is possible to provide a definite answer to this question by knowing the ratio between the radius of the disks to the superconducting coherence length, $R_{d} / \xi$ and the number of vortex-antivortex pairs generated by the disks. We have estimated $\xi(0) \sim 30 \mathrm{~nm}$, from the temperature dependence of the upper critical field and for the temperature at which the image shown in Fig. 3(e) was acquired, we obtain $R_{d} / \xi \sim 10$. In Ref. [24] it has been shown that for this ratio and considering that a maximum of three vortex-antivortex pairs is induced by the disks, the vortices on top of the disks bear a single-quantum flux unit and remain physically separated.

The penetration of extra antivortices generated by a negative external field [Fig. 3(e)] leads to an increase of 
the population of interstitial antivortices while producing no change on the vortices located on top of the disks. Interestingly, for positive fields [Fig. 3(f)], a completely different scenario is observed. Indeed, the vortices induced by the external field not only annihilate the antivortices next to the disks but also increase the occupation number of the magnetic disks close to the border of the patterned area. This indicates that for parallel alignment between the external field and the magnetic moment of the disks, two different gradients of vortex distribution coexist. On the one hand, a gradient of interstitial vortices, going from vortices at the border of the pattern, through no interstitial vortices and eventually interstitial antivortices deeper into the pattern. On the other hand, there is a gradient of the number of vortices on top of the disks, and the gradient is larger at the border of the pattern and progressively diminishes for disks located further inside the patterned area. This image reveals a composite complex critical state already envisaged in Ref. [5].

\section{ENHANCEMENT OF THE VORTEX TRAPPING BY MAGNETIZED DISKS}

In order to clearly demonstrate that for large magnetic moments a magnetic disk can trap more than three vortices, we count the number of vortices sitting at interstitial positions and compare it with the number of flux quanta on top of the disks, as the field is increased. This experiment is carried out after a cooling procedure at a nonzero field in order to avoid inhomogeneous vortex distributions and to ensure maximum repeatability irrespective of the chosen scanning spot. Figures 5(a) and 5(b) show the number of pinned and interstitial vortices, respectively, as a function of flux quanta per unit cell. For zero external field, three flux quanta sit on top of the micromagnets and correspondingly, three antivortices lie next to them. As the number of vortices per unit cell is increased, the number of interstitial antivortices decreases [Fig. 5(b)] whereas the vorticity on top of the disk remains constant. The perfect annihilation condition, i.e., for an external field inducing three vortices per unit cell, corresponds to the onset of a field interval where no interstitial vortices appear [grey area in Figs. 5(a) and 5(b)]. This existence of this so called "nulled state" has been identified in Ref. [17] for the case of magnetic disks of different diameters. Because of flux conservation, a constant number of interstitial vortices implies an increment of vorticity on top of the disks as the external field is ramped up. As shown in Fig. 5(a), for two different magnetic disks (red and yellow dots), this quantized increment of the field reaches seven trapped vortices, before new interstitial vortices, now of positive polarity, appear.

Unavoidable differences in the properties of each individual disk lead to a finite-width distribution of the number of trapped vortices per disk [28]. This is clearly demonstrated by the field profile [Fig. 5(d)] along the white dashed line depicted in Fig. 5(c), crossing two neighboring disks. Figure 5(d) shows that as the external field is
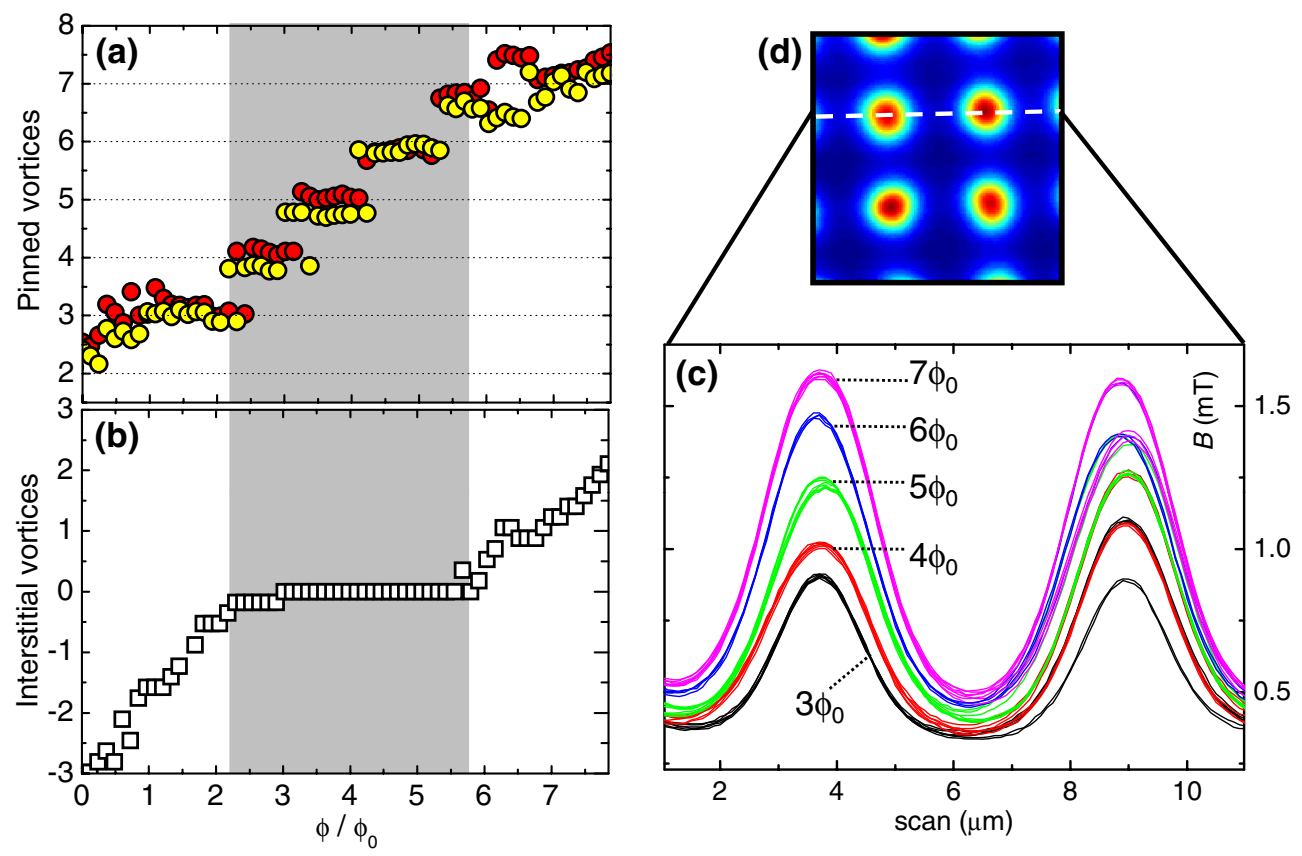

FIG. 5. Maximum number of trapped vortices by a magnetic disk. (a) Average number of vortices sitting on top of the magnetic disks as a function of field. The disks can trap up to seven vortices. (b) Average number of interstitial vortices located in between the magnetic disks. The disks are able to generate three vortex-antivortex pairs. (c) A field profile along the white dashed line is shown in panel (d). The observed discrete flux jumps reveal the trapping of extra vortices. 
continuously increased the local field exhibits jumps separated by one flux quantum. In Fig. 5(d) the field-profile curves have been grouped in such a way that a constant number of flux quanta in the left disk has the same color. This allows us to see that the right disk leads in the trapping of vortices by an extra fluxon until reaching $6 \phi_{0}$, and lags behind when switching to $7 \phi_{0}$. This finding evidences a very sharp distribution of trapped vortices per disk with a width of about one flux quanta.

\section{DIFFERENT VORTEX AND ANTIVORTEX MOBILITY}

It has been suggested in the literature [29] that spatialconfinement effects can lead to a different mobility between vortices trapped by the magnetic disks and the antivortices attached to them. For instance, for one single vortex-antivortex pair, the high degeneracy in the position of the antivortex makes it more susceptible to small excitation forces than the vortex pinned by the disk. This hypothesis can be experimentally demonstrated by using a scanning ac-susceptibility technique, which reveals the points of maximum amplitude of oscillation of each individual vortex when submitted to an external ac field [30]. In Fig. 6(a) the SHPM images obtained at zero field and with the disks magnetized shows the presence of 7 antivortices, 3 at the center and 4 at the rims of the scanning area. This vortex configuration is then excited with a small ac field and the locations of maximum oscillation of each individual vortex is recorded by the scanning acsusceptibility image shown in Fig. 6(b). This technique shows that two of the central antivortices oscillate between (a)
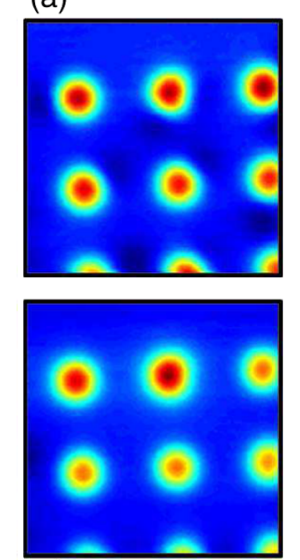

(d) (b)

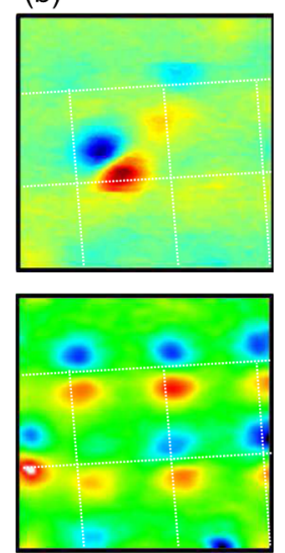

(e) (c)
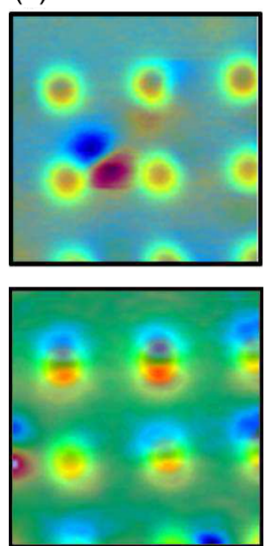

$(f)$
FIG. 6. Probing the mobility of vortex-antivortex pairs. (a), (d) Scanning Hall Microscopy images at $H=0$ and $T=6.9 \mathrm{~K}$ for two different magnetic moments of the disks. (b), (e) Scanning ac-susceptibility images taken with $h=0.2 \mathrm{Oe}$ and $h=0.6$ Oe, respectively, and a frequency of excitation $77 \mathrm{~Hz}$. Panels (c) and (f) show a superposition of panels (a) and (b), and (d) and (e), respectively, in order to unambiguously identify the vortices susceptible to movement. bistable points whereas no motion is detected for any vortex sitting on top of the magnetic disks. In Fig. 6(c) the two panels, (a) and (b), have been superimposed to unambiguously identify the vortices susceptible to move. It is important to note that this technique is able to detect only periodic motion between two points. Therefore, the lack of signal associated with the rest of the antivortices can be either because they remain pinned or due to a more nonperiodic trajectory during the ac excitation. By increasing the amplitude of the ac excitation eventually we are able to shake also the vortices on top of the disks. This is shown in Figs. 6(d)-6(f), where, for the sake of clarity, a lower magnetic moment with only one antivortex present at the left side of the scanning area, has been chosen.

\section{CONCLUSION}

Our measurements reveal the microscopic static and dynamic behavior of vortex-antivortex pairs spontaneously induced by micromagnets in close proximity to a superconducting film. By adjusting the average magnetic moment $M$ of the micromagnets it is possible to quantify the number of vortices that a single magnetic disk can trap for different magnetizations and applied fields. Unlike superconducting films with no magnetic templates, the penetration of vortices from the border of the pattern leads to the coexistence of two graded vortex distributions, one on top of the micromagnets and another formed by interstitial vortices. These studies provide important new information for the investigation of discretized quantum forces in levitating micromagnets and for the microscopic mechanism occurring when magnetic pinning centers are introduced in superconducting films.

\section{ACKNOWLEDGMENTS}

This work was supported by Methusalem funding by the Flemish government, the Flemish Science Foundation (FWO-Vl), the Belgian Science Policy, and the ESF NES network. A. V.S. and W. G. acknowledge support from FWO-Vl. The authors are thankful to Bart Raes and Jo Cuppens for useful discussions and the critical reading of the manuscript.

[1] V. Arkadiev, A Floating Magnet, Nature (London) 160, 330 (1947).

[2] S. Erdin, I. F. Lyuksyutov, V. L. Pokrovsky, and V. M. Vinokur, Topological Instability in a Ferromagnet Superconducting Bilayer, Phys. Rev. Lett. 88, 017001 (2001).

[3] M. Lange, M. J. Van Bael, Y. Bruynseraede, and V. V. Moshchalkov, Nanoengineered Magnetic-Field-Induced Superconductivity, Phys. Rev. Lett. 90, 197006 (2003).

[4] Z. Yang, M. Lange, A. Volodin, R. Szymczak, and V. V. Moshchalkov, Domain-Wall Superconductivity in Superconductor-Ferromagnet Hybrids, Nature Mater. 3, 793 (2004). 
[5] D. G. Gheorghe, R. J. Wijngaarden, W. Gillijns, A. V. Silhanek, and V.V. Moshchalkov, Magnetic Flux Patterns in Superconductors Deposited on a Lattice of Magnetic Dots: A Magneto-Optical Imaging Study, Phys. Rev. B 77, 054502 (2008).

[6] Nuria Del-Valle, Carles Navau, Alvaro Sanchez, and Du-Xing Chen, Tunability of the Critical-Current Density in Superconductor-Ferromagnet Hybrids, Appl. Phys. Lett. 98, 202506 (2011).

[7] S. B. Haley, Length Quantization in Levitation of Magnetic Microparticles by a Mesoscopic Superconducting Ring, Phys. Rev. Lett. 74, 3261 (1995).

[8] S. B. Haley and H. J. Fink, Quantized Levitation States of Superconducting Multiple-Ring Systems, Phys. Rev. B 53, 3497 (1996).

[9] J. C. Wei, L. Horng, and T. J. Yang, Magnetic Force Signal of Vortex Creation in Type-II Superconducting Thin Films, Physica C (Amsterdam) 280, 311 (1997).

[10] O. M. Auslaender, L. Luan, E. W. J. Straver, J. E. Hoffman, N. C. Koshnick, E. Zeldov, D. A. Bonn, R. Liang, W. N. Hardy, and K. A. Moler, Mechanics of Individual Isolated Vortices in a Cuprate Superconductor, Nature Phys. 5, 35 (2008).

[11] U.S. Department of Energy, Basic Research Needs for Superconductivity: Report of the Basic Energy Sciences, (2006), http://science.energy.gov/bes/news-and-resources/ reports/basic-research-needs/.

[12] A. Yu. Aladyshkin, A. V. Silhanek, W. Gillijns, and V. V. Moshchalkov, Nucleation of Superconductivity and Vortex Matter in Superconductor-Ferromagnet Hybrids, Supercond. Sci. Technol. 22, 053001 (2009).

[13] S. C. Wimbush, J.H. Durrell, R. Bali, R. Yu, H. Wang, S. A. Harrington, and Judith L. MacManus-Driscoll, Practical Magnetic Pinning in YBCO, IEEE Trans. Appl. Supercond. 19, 3148 (2009).

[14] A. Palau, H. Parvaneh, N. A. Stelmashenko, H. Wang, J. L. Macmanus-Driscoll, and M. G. Blamire, Hysteretic Vortex Pinning in Superconductor-Ferromagnet Nanocomposites, Phys. Rev. Lett. 98, 117003 (2007).

[15] F. Hellman, E. M. Gyorgy, D. W. Johnson, Jr., H. M. O'Bryan, and R.C. Sherwood, Levitation of a Magnet over a Flat Type-II Superconductor, J. Appl. Phys. 63, 447 (1988).

[16] E. H. Brandt, Levitation in Physics, Science 243, 349 (1989).

[17] J. S. Neal, M. V. Milošević, S. J. Bending, A. Potenza, L. San Emeterio, and C. H. Marrows, Competing Symmetries and Broken Bonds in Superconducting Vortex-Antivortex Molecular Crystals, Phys. Rev. Lett. 99, 127001 (2007).
[18] W. B. Zeper, F. J. A. M. Greidanus, P.F. Carcia, and C.R. Pincher, Perpendicular Magnetic Anisotropy and Magneto Optical Kerr Effect of Vapor-Deposited Co/Pt-Layered Structures, J. Appl. Phys. 65, 4971 (1989).

[19] W. Gillijns, A. V. Silhanek, and V. V. Moshchalkov, Tunable Field-Induced Superconductivity, Phys. Rev. B 74, 220509(R) (2006).

[20] A. Hoffmann, P. Prieto, and I. K. Schuller, Periodic Vortex Pinning with Magnetic and Nonmagnetic Dots: The Influence of Size, Phys. Rev. B 61, 6958 (2000).

[21] D. J. Morgan and J.B. Ketterson, Asymmetric Flux Pinning in a Regular Array of Magnetic Dipoles, Phys. Rev. Lett. 80, 3614 (1998).

[22] M. J. Van Bael, M. Lange, S. Raedts, V. V. Moshchalkov, A. N. Grigorenko, and S. J. Bending, Local Visualization of Asymmetric Flux Pinning by Magnetic Dots with Perpendicular Magnetization, Phys. Rev. B 68, 014509 (2003).

[23] M. V. Milošević and F. M. Peeters, Interaction Between a Superconducting Vortex and an Out-of-Plane Magnetized Ferromagnetic Disk: Influence of the Magnet Geometry, Phys. Rev. B 68, 094510 (2003).

[24] M. V. Milošević and F. M. Peeters, Superconducting Wigner Vortex Molecule Near a Magnetic Disk, Phys. Rev. B 68, 024509 (2003).

[25] W. Gillijns, M. V. Milošević, A. V. Silhanek, and V. V. Moshchalkov, F. M. Peeters, Influence of Magnet Size on Magnetically Engineered Field-Induced Superconductivity, Phys. Rev. B 76, 184516 (2007).

[26] D. J. Priour, Jr. and H. A. Fertig, Vortex States of a Superconducting Film from a Magnetic Dot Array, Phys. Rev. Lett. 93, 057003 (2004).

[27] M. V. Milošević and F. M. Peeters, Vortex-Antivortex Lattices in Superconducting Films with Magnetic Pinning Arrays, Phys. Rev. Lett. 93, 267006 (2004).

[28] W. Gillijns, A. V. Silhanek, and V.V. Moshchalkov, Superconducting Microrings as Magnetic Pinning Centers, Appl. Phys. Lett. 91, 202510 (2007).

[29] M. Lange, M.J. Van Bael, A. V. Silhanek, and V.V. Moshchalkov, Vortex-Antivortex Dynamics and Field-Polarity-Dependent Flux Creep in Hybrid Superconductor/Ferromagnet Nanostructures, Phys. Rev. B 72, 052507 (2005).

[30] R. B. G. Kramer, G. W. Ataklti, V. V. Moshchalkov, and A. V. Silhanek, Direct Visualization of the Campbell Regime in Superconducting Stripes, Phys. Rev. B 81, 144508 (2010). 